\title{
The X-shooter spectrograph: a new concept of mechanical assembly for a multiple-arm Cassegrain Instrument
}

\author{
Niels Michaelsen ${ }^{a}$, Vincenzo De Caprio ${ }^{b, e}$, Niels Christian Jessen ${ }^{c}$, Jean-Louis Lizon ${ }^{d}$, Ruben \\ Mazzoleni $^{d}$, Marco Riva ${ }^{e}$, Paolo Spano ${ }^{e}$, Anton Norup Sørensen ${ }^{a}$, Matteo Tintori ${ }^{e}$, Dennis W. \\ Wistisen $^{a}$, Hans Dekker ${ }^{d}$, Per Kjærgaard Rasmussen ${ }^{a}$, Filippo M. Zerbi ${ }^{e}$, Sandro D'Odorico ${ }^{d}$ \\ ${ }^{a}$ Niels Bohr Institute, Juliane Maries Vej 30, 2100 Copenhagen - Denmark \\ ${ }^{b}$ INAF - IASF, Via E. Bassini 15, 20133 Milano (MI) - Italy \\ ${ }^{c}$ Danish National Space Center, Juliane Maries Vej 30, 2100 Copenhagen - Denmark \\ ${ }^{d}$ European Southern Observatory, Karl-Schwarzschild-Str. 2, 85748 Garching b. Müenchen (D) \\ ${ }^{e}$ INAF - Osservatorio Astronomico di Brera, Via E. Bianchi 46, 23807 Merate (LC) - Italy
}

\begin{abstract}
$\mathrm{X}$-shooter is a second generation VLT instrument currently under construction by a Consortium of Institutes from Denmark, Italy, The Netherlands, France and ESO. X-shooter is designed to acquire intermediate (5000-10000) resolution spectra of single objects in an unprecedented wide wavelength coverage (320-2500 nm). In order to maximize efficiency the beam is divided into 3 arms (UV, VIS and NIR) by a system of dichroics. X-shooter is designed for the Cassegrain focus of one VLT unit. The mechanical assembly has to provide specific solutions to maintain 3 arms within the strict tolerances required by the intermediate resolution, during the typical motions of the Cassegrain focal station. It must as well ensure the permanent co-alignment of the 3 slits and the stability of the spectral format on the focal plane of each arm, allowing long intervals between calibration exposures. The above requirements have been met via an innovative mechanical design merging passive stiffness and active control to obtain a light, accessible and functional assembly. This paper gives a description of the $\mathrm{X}$-shooter mechanical assembly with main emphasis on the common "backbone" structure and the UV- and VIS spectrograph arms.
\end{abstract}

\section{INTRODUCTION}

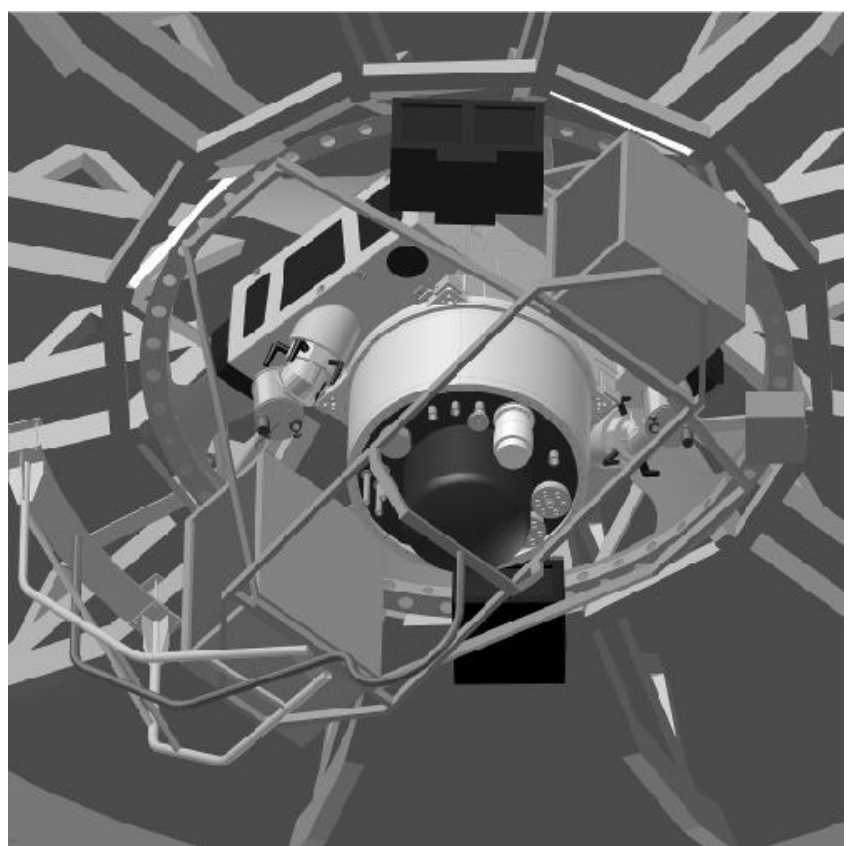

Figure 1. Overview of the X-shooter spectrograph
$\mathrm{X}$-shooter is a 3 armed UV-VIS-NIR spectrograph for the Cassegrain Focus of the VLT. A general layout is represented in Figure 1. Each of the arms is a selfconsistent instrument which will be mounted on a common "backbone" structure which contains all the beam partition and beam preparation devices. The NIR arm is cryogenic and is fully developed in a cryostat. The UV and VIS arm have instead very similar layout and have been designed in parallel in order to save engineering work and maximize the number of common parts. The electronics for the moving functions and the detectors are placed on an independent mechanical frame.

The optical design of each spectrograph is the so-called $4 \mathrm{C}$ design, and a cross-dispersed echelle spectral format is used. 


\section{THE BACKBONE AND THE CONNECTION TO THE VLT}

The Backbone provides the connection between the VLT cassegrain adaptor and the three spectrographs. At the same time the Backbone houses a number of common functions such as the calibration system, a focal plane unit with the associated target acquisition camera, and an optical table housing the dichroics splitting the light into the three spectrograph arms together with the atmospheric dispersion correctors and the piezo driven flexure compensation system.

The main Backbone structure is a welded box in aluminum with a size of about $1 \mathrm{~m}^{3}$ and a total weight of $680 \mathrm{~kg}$. On top of this structure a steel ring is mounted as interface to the VLT cassegrain adaptor, see Figure 2 below. Steel has been chosen in order not to impose radial stress on the rotator bearing of the cassegrain adaptor due to thermal load. The main aluminum structure is a double walled box. The inner "box" consists of a $20 \mathrm{~mm}$ base plate on which a cylinder centered with the optical axis is placed. On the four sides of this cylinder 4 hollow corner legs are placed. Four outer plates then close the box. All wall plates are in $18 \mathrm{~mm}$ AlMg4.5Mn. Two cylindrical pieces are welded in at the center of two opposite walls for common access. Two conical pieces are welded in at the other two opposite walls for the two optical spectrographs. HeliCoils are mounted in the three spectrograph mounting holes. The UVB and VIS spectrographs will be mounted on two opposite sides. The NIR spectrograph will be mounted below the bottom base plate.
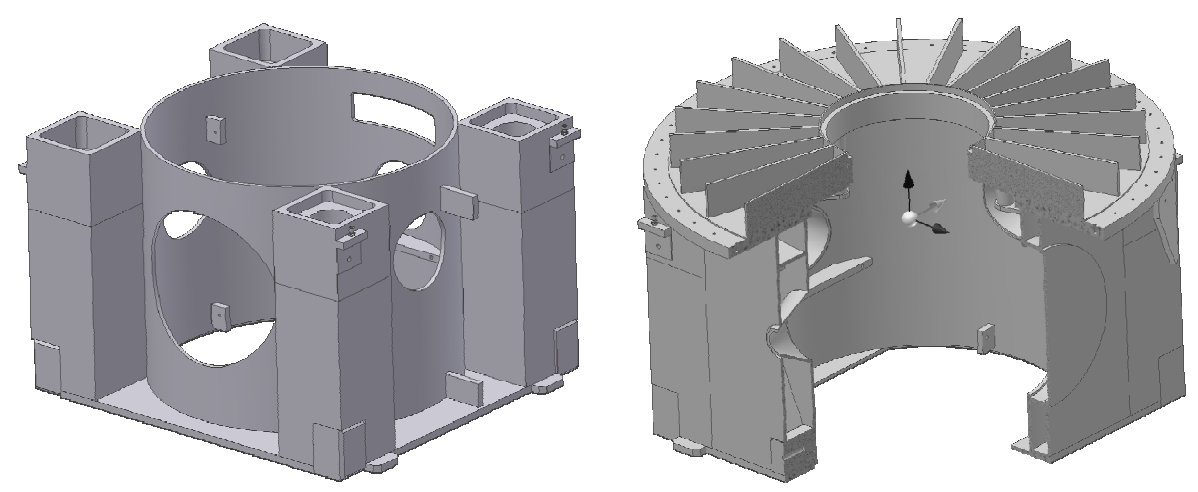

Figure 2 Bottom Backbone plate with the central cylindrical piece and the four hollow corner legs (left) and a three-quarter view of the welded aluminum Backbone without the top steel ring (right).

\section{THE COMMON FUNCTIONS IN THE BACKBONE}

\section{The SD40-2 servo drive unit}

$\mathrm{X}$-shooter (including the Backbone) contains in total 13 motor driven movements of which 6 are linear and 7 are rotational movements. All movements are driven by the same "SD40-2" Servo-drive unit. This unit is developed to match the X-shooter moving functions and rely on a previous ESO design. On the top of a Maxon A-max 32 DC motor a planetary gearbox $(1: 14)$ is mounted together with a tacho at the rear end (see Figure 3). A slide clutch protects the motor if the limit switch fails or the movement is blocked. The moment is transferred through an internal gear to an output axle. A Heidenhain ROD1020 encoder is mounted directly on the opposite end of this output axle. Two connectors connect the SD40-2 to the electronics controller and to the initialization and limit switches.

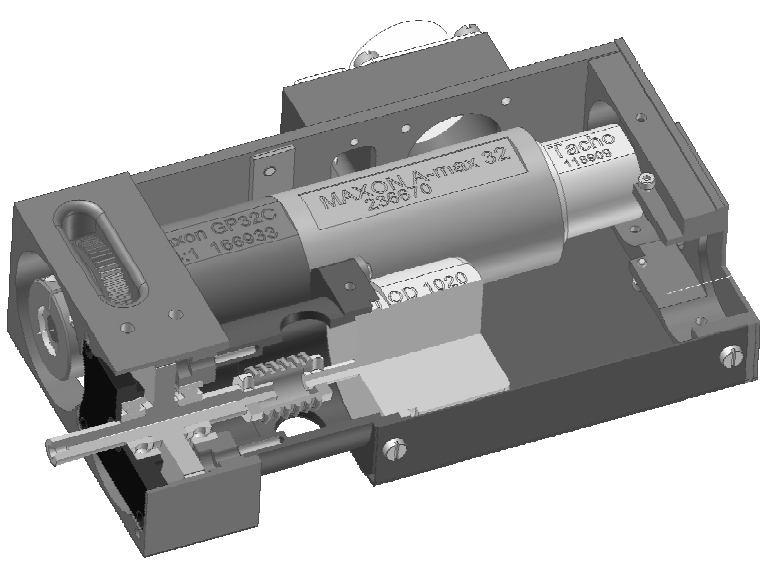

Figure 3 A SD40-2 unit with a side plate removed 


\section{The Instrument Shutter and the Calibration Lamps Unit}

Below the top steel ring a rotating butterfly shutter is placed to protect the instrument from dust and also to shut out light during day-time calibrations. The shutter is driven by the SD40-2 servo drive unit. The Calibration system is mechanically divided into three sub systems: Two different lamp units which are mounted outside of the backbone, a dual mirror carriage which is mounted on the instrument shutter base plate and a dual relay lens support which is mounted on one of the three support struts for the A\&G unit. The lamp units are shown rightmost, (Figure 4) attached to a plate which matches the outer wall of the backbone. The dual relay lenses are shown schematically as black rings directing the beams. The calibration mirror carriage is placed below the entrance shutter, reflecting a calibration beam to the Cassegrain focus.

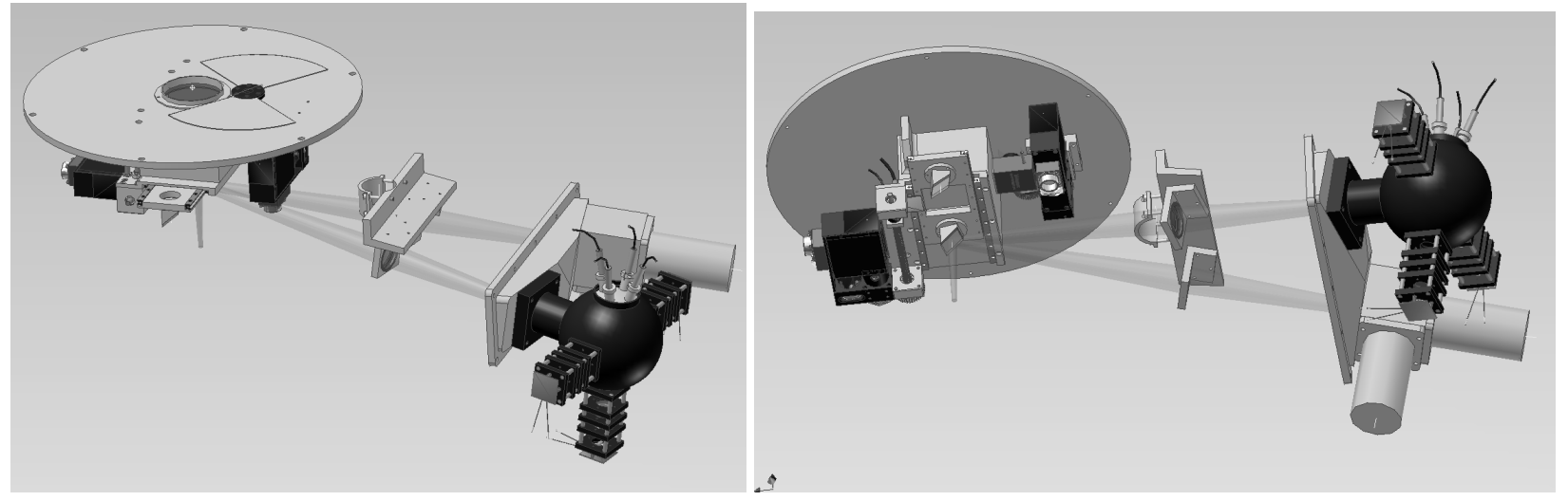

Figure 4 Instrument shutter and calibration lamps unit viewed from above (left) and below (right)

\section{The Acquisition and Guide Unit}

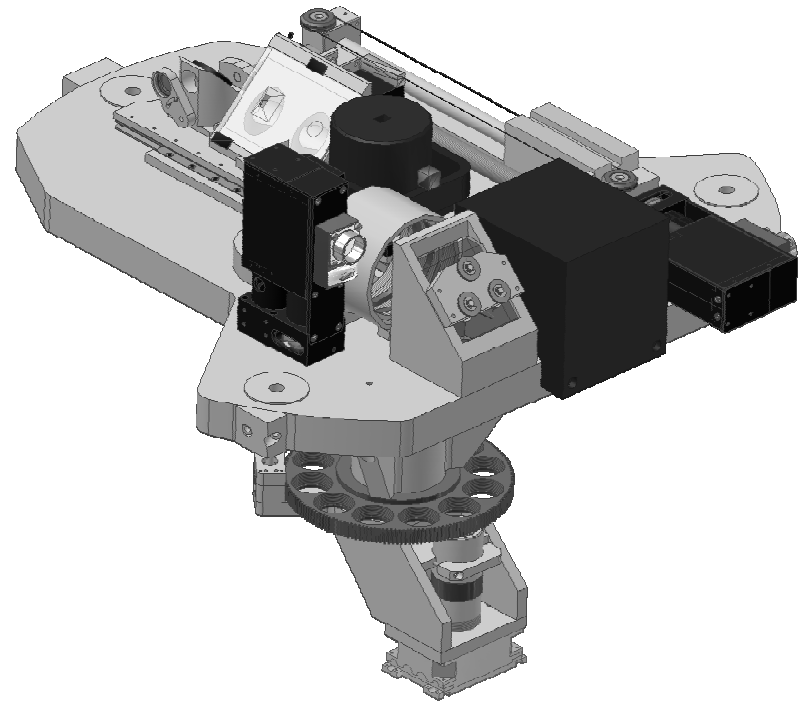

Figure 5 Isometric view of the A \&G Unit
The telescope's Cassegrain focal plane falls inside the Backbone. At this position the so-called Acquisition and Guide unit is placed. Here one has the mirror carriage (see Figure 5) with 5 positions. One position is open for regular science observations, one houses an integral field unit, another a mirror directing the light to the acquisition camera. The remaining two positions are used for slit viewing and for a pinhole that serves as alignment reference. For alignment, the mirror slide must be positioned with a precision of $\pm 0.56 \mu \mathrm{m}$. The preloaded mirror carriage moves on high precision SKF rails. The carriage is driven by the SD40-2 servo drive unit in a $190 \mathrm{~mm}$ range at $3.6 \mathrm{~mm} / \mathrm{sec}$. The light to the acquisition camera passes the first folding mirror before it enters the big collimator lens, then the light is directed downwards at the next folding mirror and passes the filter wheel before it enters the optical camera after which it forms an image on the CCD camera. The filter wheel is moved by another identical SD40-2 unit. All units are mounted on a $40 \mathrm{~mm}$ thick and light weighted base plate which is fixed to the walls of the Backbone at three points. 


\section{The Common Pre-slit Table}

The light is split up into the three beams and directed to the respective spectrograph arms in the unit called "the common pre-slit table" shown in Figure 6. The dichroics are placed in the compact box structure in the middle of the vertical optical table. Blue light is reflected on the first dichroic to a folding mirror which directs the light to the right. The tip-tilt of the folding mirror can be controlled by three piezo actuators. In this way mechanical flexure inside the Backbone can be compensated. After the folding mirror light passes two barrels with prisms and lenses which compensates the effect of atmospheric dispersion and at the same time changes the F-ratio of the incoming beam and also re-image the Cassegrain image plane on the slit of the blue spectrograph. The mechanics for the atmospheric dispersion corrector is rather delicate. Each ADC barrel must be centered on the optical axis to within $\pm 15 \mu \mathrm{m}$ to make certain that image wobble from $\mathrm{ADC}$ rotation during an exposure remains acceptable. During an exposure, the rotation angle of the ADC must be updated regularly with a precision of 1 arcminute. The two barrels are mounted and aligned by SKF rails in a common frame. The rotation is accomplished using the rotational ring as a gear-wheel driven by the SD40-2 unit through a spring preloaded belt. The radial accuracy of the rotational ring is ensured by 2 SKF FPAB 400 bearings. The movement along the optical axis is a one time adjustment done by screws. The second dichroic reflects the visual light to the piezo-driven folding mirror and on to the atmospheric dispersion corrector for the visual light. This system is identical to the blue system from a mechanical point of view. Finally the infrared light passes straight through the two dichroics to a piezo controlled folding mirror and then on the in NIR spectrograph. Here no atmospheric dispersion corrector is used.

The preslit optical table is stiffened by a triangular envelope kind of structure on the backside of the table. Also the four SD40-2 servo-drive units are mounted on the backside of the table.

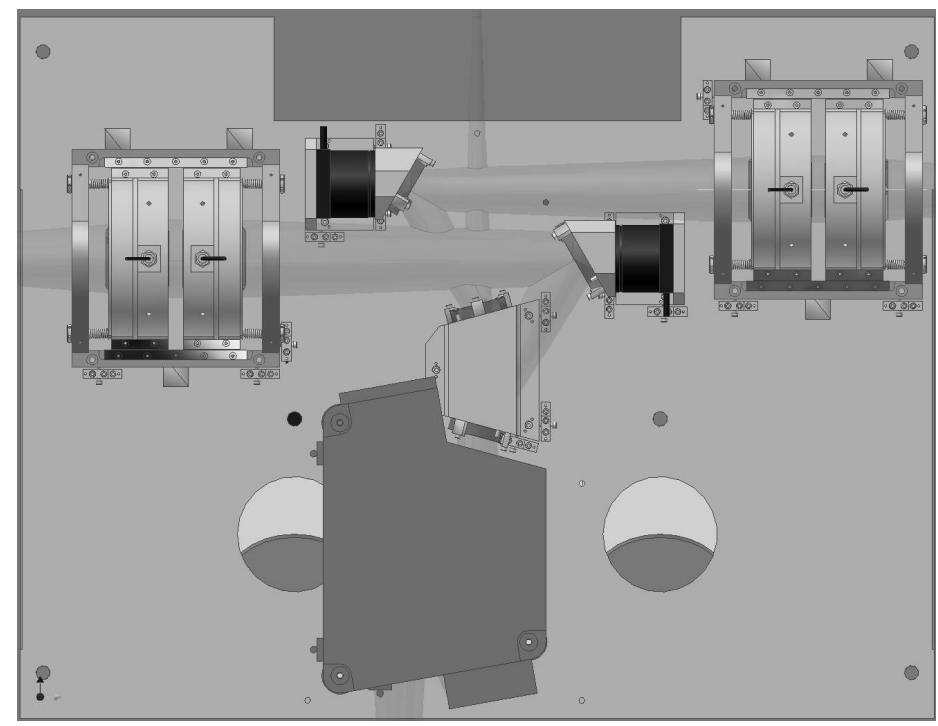

Figure 6 The common pre-slit table with the box for the two dichroics (middle), the three piezo units (black), the two atmospheric dispersion correctors, and the NIR pre-slit box.
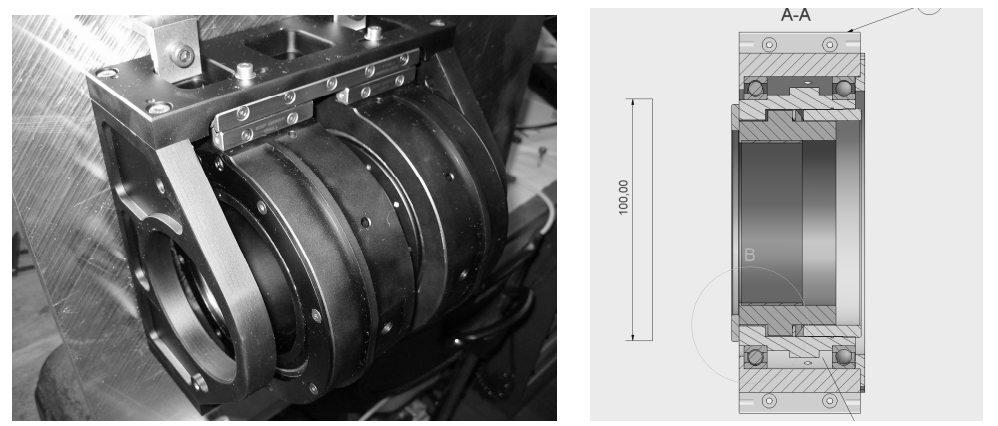

Figure 7 The atmospheric dispersion corrector. The proto-type (left) and the design of one unit (right) showing the rotating system. 


\section{The Bench}

\section{THE UV-B AND VIS SPECTROGRAPHS}

The benches of the UV and VIS spectrographs are almost identical. A bench consists of two main parts which both are made in aluminum (AlMg4.5Mn). The first part is the bottom plate, which is milled out of one piece. This part contains the mounting interface to the Backbone on the one side, and provides the mounting bench on the other side for most of the optical supports. The second part of the bench is a box structure with two internal walls and a cylindrical piece with the mounting flange for the CCD cryostat. One of the walls carry the holder for the main mirror, the other wall stiffens the structure at the cylindrical piece. The second part is welded together. The two parts are bolted and glued together before the final milling of the internal surfaces. The overall dimensions of the benches are $\left(\mathrm{W}^{*} \mathrm{H} * \mathrm{D}\right) 1300 * 580 * 450 \mathrm{~mm}$. The wall thickness is designed to be $12 \mathrm{~mm}$ all over and the weight of the whole structure is about $125 \mathrm{~kg}$.

Basically each bench is mounted directly on the sides of the Backbone by three M16 bolts. A fourth point is added in form of a $\varnothing 12 \mathrm{~mm}$ aluminum rod placed where the gravitational induced stress is highest. Three adjustable hard points on the bench with mating reference points on the Backbone ensures the reference between the bench and the Backbone.

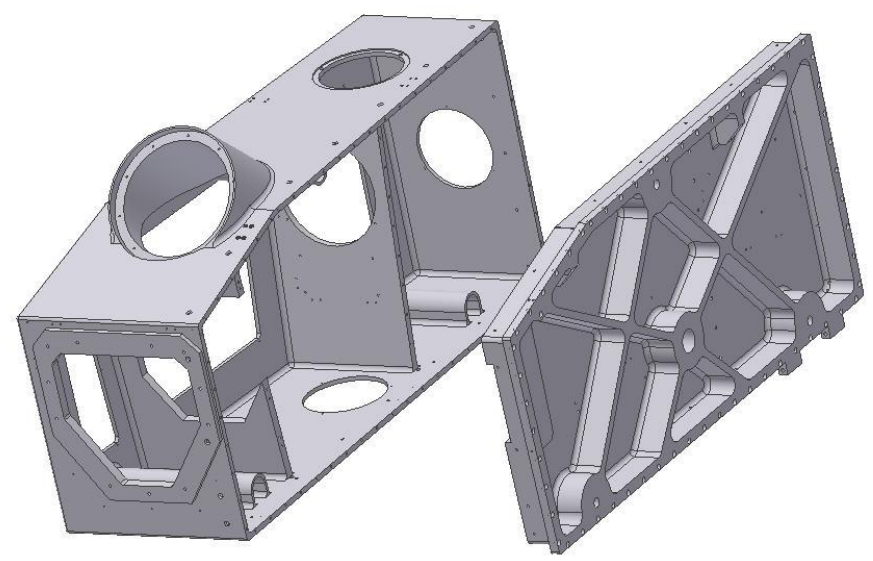

Figure 8. The two main parts of a bench

\section{THE MECHANICAL SUPPORTS FOR THE OPTICS}

The two spectrographs present a very similar optical design. This characteristic is very useful, because in this way it is possible to simplify the mechanical design of similar elements. We have decided to twin the conceptual design for each optical element in order to balance the weights of the two arms and to simplify the design operation. Many of the optical elements will be glued to invar or steel pads. In the preliminary test phase we plan to use the $3 \mathrm{M}^{\circledR}$ "Scotch-Weld 2216 $\mathrm{B} / \mathrm{A}$ " epoxy adhesive. Following the needs of the alignment procedures and adjusting device, we have decided to design a standard support adaptable for most of the optical elements. This support is sketched out in the panels of Figure 9.

There are two types of support designed with the same philosophy. The first one is used when the support wall in the bench lies in correspondence of the front face of the optical element, i.e. the Main Mirror. The second one is used when the optical element is far away from the bench (or the wall of the bench); in this case the optics are mounted on a structure that transfers all the loads to the bench: it is the case of the Grating and the Pupil Mirror.

There is an invar or steel spherical pad glued to a surface of the optical element. In the pad there is a cylindrical cave in which a lubricant graphite tube is inserted. In the pad a steel shaft is inserted with a spherical head, which is leaned onto a cone derived from the tube. 

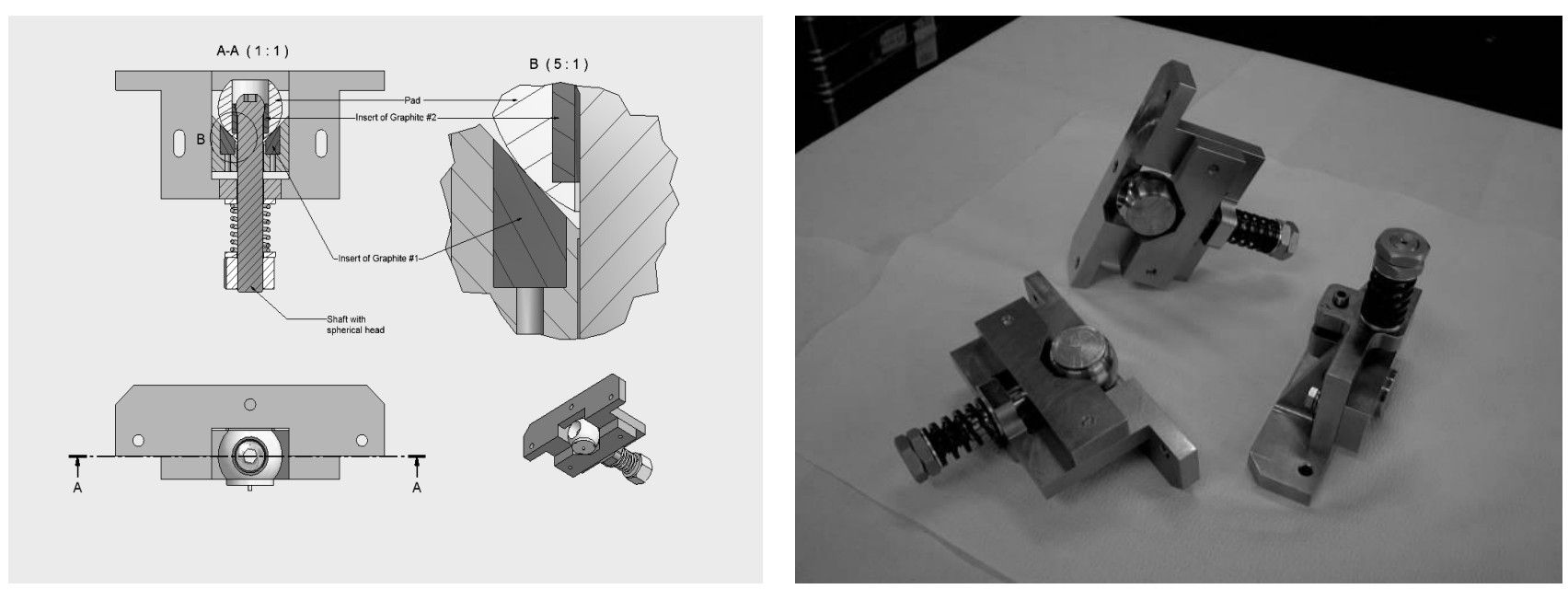

Figure 9 Overall view of the adaptable support.

The external surface of the pad is supported by a V-shaped plate ${ }^{1}$. The movement of the pad in the V-Shape is like a spherical joint. The plate can shift along the shaft axis direction. The movement is given by a grain which forces a tongue on a plate fixed on the aluminum support (frame or castles). The fixed plate works as a guide for the shifting of the mobile plate; its structural function is to support the mobile plate via two screws that are locked after the alignment procedure.

The support has to absorb the differential extension between the glass element and the aluminum support, for this reason the plates have a hole in which is inserted the shaft. The hole is quite bigger than the shaft. The hole in the mobile plate has a co-axial ring near the V-shape. The ring combined with the V-shape realize a cart constraint with the free Degree Of Freedom (DOF) normal to the glued surface. The shaft is locked via a nut. Between the nut and the plate there is a spring, which is useful in the alignment phase because it guarantee the rollback of the pad, otherwise its main function is to absorb the thermal stresses.

\section{The Slit Carriage}

The entrance slit is selected among predefined slits in a slit plate. This plate is mounted in the Slit Carriage which is mounted on SKR high precision rails. The carriage is driven by a SD40-2 unit. The iris-type exposure shutter is mounted on top of the slit carriage. Also this unit carries a field lens and the first folding mirror (Figure 10). Important design parameters are the accuracy of the movement of the slit carriage (reproducibility \pm 0.02 " corresponding to $\pm 0.6 \mu \mathrm{m})$ and vignetting constraints.

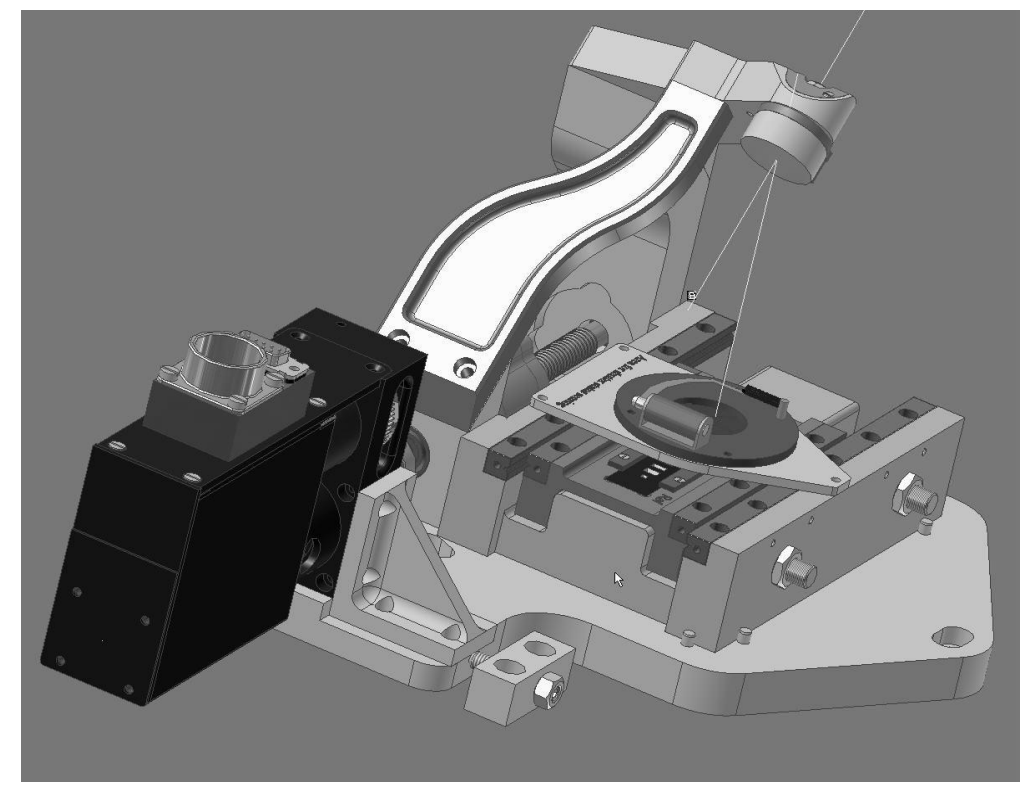

Figure 10 Overall layout of the slit carriage

\footnotetext{
${ }^{1}$ A graphite wedge is inserted in the plate beside the contact area..
} 


\section{THE MAIN MIRROR}

The supports of the main mirror (Figure 11) adopt the same philosophy of the "standard supports". They are collected on a frame, used also as alignment tool. This frame has a shape which follows the perimeter of the chamfered Main Mirror with a thickness of $19 \mathrm{~mm}$. Each support is connected to the frame via three $M 6$ screws. The frame instead is fixed to the bench via 10 eyeleted holes, in this way it is possible to rotate the frame with the whole Main Mirror support system, in order to adjust the de-centre (DX, DY) and the tilt (TZ) as required in the optical alignment procedure.
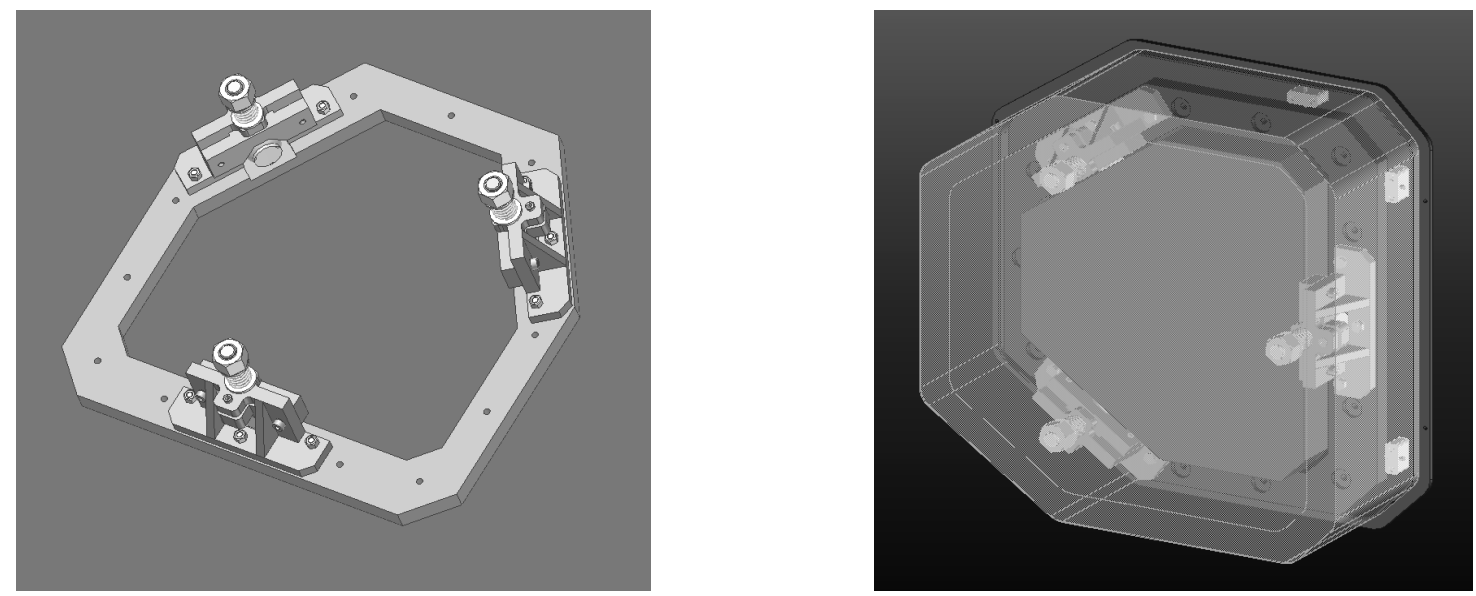

Figure 11 Overall view of the Main Mirror Support.

The main mirror has been chamfered in a trapezoidal shape following the beam footprint. This choice allows an appreciable lightening of the main mirror.

\section{THE PRISM/GRATING COMBO UNIT}

The prism and the grating are mounted on the same plate (Footprint Plate - thickness $5 \mathrm{~mm}$ ) which will be fixed at the bench via six $M 5$ screws. This choice allows to enlarge the footprint of the two subunits and consequently distributes the weight of prism and grating opto-mechanical elements on a bigger surface, reducing the stresses induced to the bench. In this way it is possible to align those sub-units out of the bench and then insert them avoiding problems due to the accessibility of the alignment tools. The Footprint Plate is designed in order to have two perpendicular edges used for the reference bars. In Figure 12 are shown the Combo Unit and one of the three pads required to bond the Prism on the mobile base of the support.
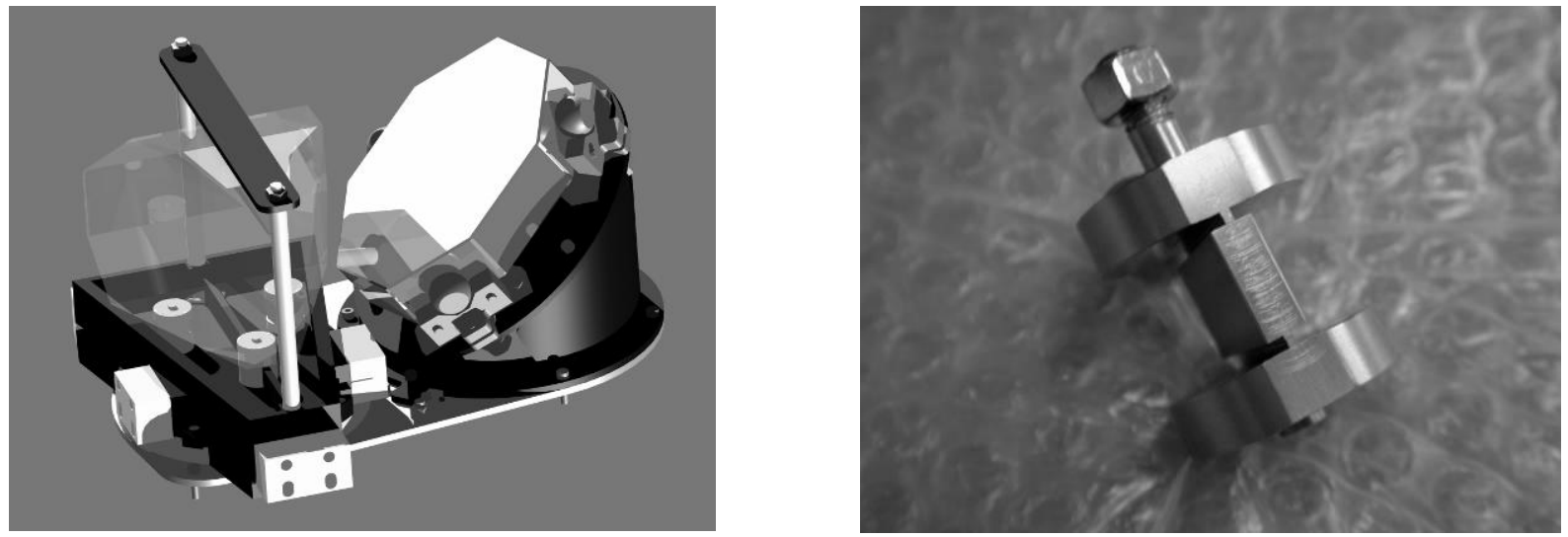

Figure 12 Overall view of the Combo Unit and one pad of the Prism. 
The prism is handled via three Macor pads glued to its bottom surface. They are bound on a triangular plate (Mobile Plate). This plate is linked to a twin one (Fixed Plate) via two rounded plates (front and behind), which act as a guide for the tilting movement. Two other lateral plain plates are used in order to reach the required stiffness of the whole support. Between the twin plates there is a cylinder which acts as a pin for the required tilt of the prism. This regulation is controlled by a micrometer screw placed behind the prism on the symmetrical plane.

There are two shafts orthogonal to the MbP which handle a cover plate. This plate has two functions; the first one is to fix a silicone disk useful in case of failure of a glued pad. Another function is to support a handle used for alignment and maintenance operations. The whole support is fixed to a collar screwed on the Footprint Plate ${ }^{2}$; this connection allow a small tilt in the dispersion plane (as required from optical specifications).

The materials used will be: anodized aluminum for the whole subunit except for the Macor pads and the pin, the screws, the nuts and the silicone diskette. The plates used for the connection of the Fixed Plate and the Mobile Plate will be in glassfiber coupled with epoxy resin. The micrometer screw will be in stainless steel.

The echelle-grating, which has an octagonal shape, is handled via three pads on its lateral surfaces. The three pads are components of the adaptive supports that are screwed on an elliptical plate (Top Elliptical Plate) that in turn it is bound to a cylinder. The Cylindrical Base positions the grating in the correct position and it gives the correct stiffness to the support. The lower part of the Cylindrical Base has a frame with six drilled holes. Three of these are used to bond it to the Footprint Plate and the remainders holes are co-axial with three holes in the Footprint Plate used to connect the combo unit with the bench. The Cylindrical Base and Top Elliptical Plate are made in anodized aluminium, the other parts in steel and invar.

\section{THE PUPIL MIRROR}

The Pupil Mirror is mounted on a structure made by aluminum 6060 using the "standard small support" described above. The aluminum machined structure provides the angle needed between the Pupil Mirror (back surface) and the wall on the bench that accommodates the corrector lens. This structure is designed in order to allow the mounting-dismounting of the Pupil Mirror uncoupled from the Corrector Lens. The aluminum structure, after the machining, will undergo an anodizing process. On the back-side the support is screwed, with four M5 on its corners, to the Lens Wall of the Bench. A compared view of the Pupil Mirrors supports is given in Figure 13.
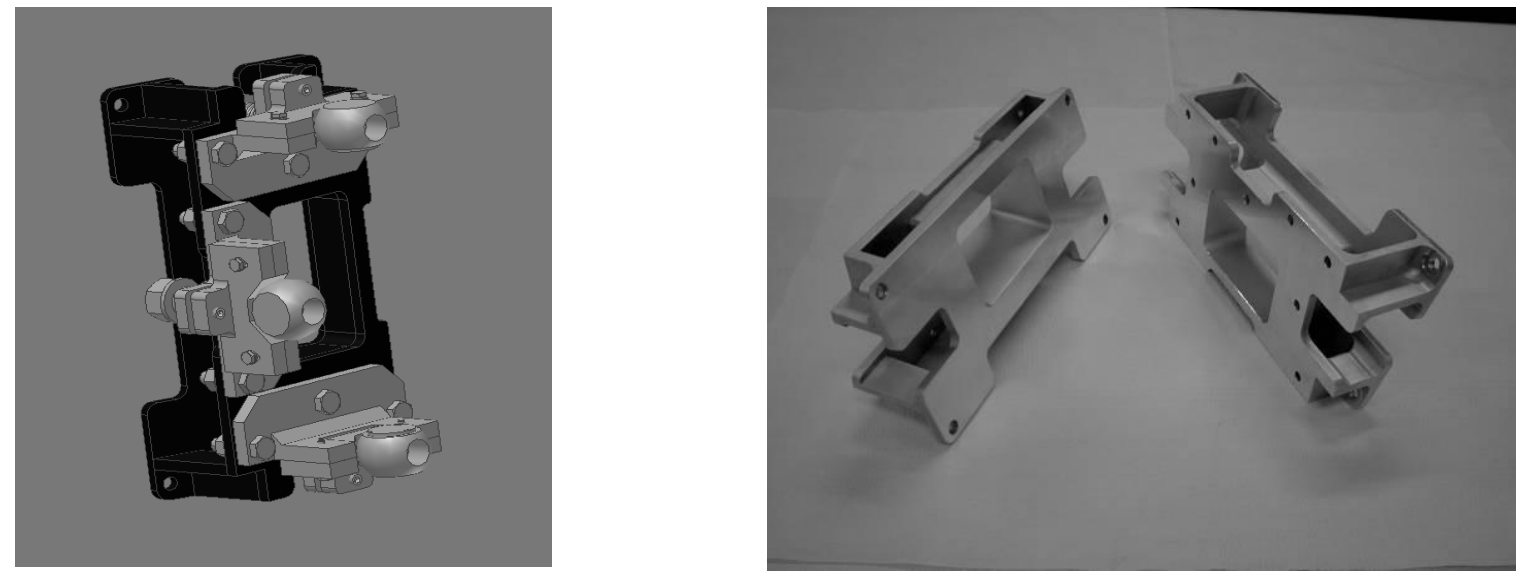

Figure 13 Overall view of the support of the Pupil Mirror.

\section{THE CRYOSTAT, THE CAMERA AND THE AUXILIARY MECHANICHAL INTERFACES}

\footnotetext{
${ }^{2} \mathrm{FtP}$ is the footprint plate used for the connection of the prism and the grating in a Combo-subunit.
} 
The Cryostats both for the UV and the VIS spectrographs will be manufactured at ESO and delivered pre-mounted to the integration site at Merate Observatory. The CCD Detector head shown in Figure is made of aluminum. Between the Cryostat Head and the LN2 container a wedge, with an internal angle of $+45 \mathrm{deg}$, is mounted. The wedge is a part of the vacuum system and carry the LN2 container almost vertical when the telescope is pointing to zenith.

The flange of the Cryostat is fitted with three $(3 \mathrm{~mm} \mathrm{H7})$ slots which allow to reproduce the position within a few microns. The chip will be centered on the mechanical center of the flange within an accuracy of \pm 200 microns, along the optical axis the chip will be position at $2.5 \pm 0.010 \mathrm{~mm}$ from the flange. The parallelism of the chip will be $\pm 0.010 \mathrm{~mm}$ compared to the flange.

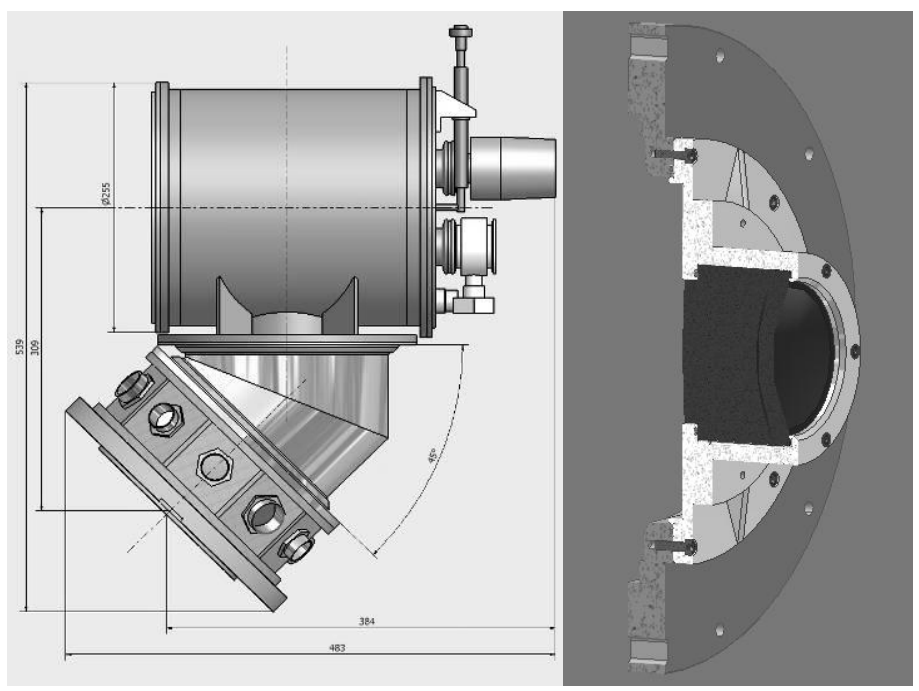

Figure 14 Overall view of the Cryostat and the Vacuum Barrel.

The vacuum sealing on the cryostats will be guaranteed by the last lens of the Camera train that will be purposely shaped. The shapes of the window Cryostat (named "Vacuum Barrel") are given in Figure 14.

The vacuum sealing is obtained via a Viton O-ring (30\% compression) positioned between the last lens of the doublet (VIS case) or the last lens (UV case) and the back face of the "Vacuum Barrel". A retaining flange, on the front face of the lens, maintains the initial position of the camera lens before the vacuum is made. The surface on the back face of the barrel, is not anodized (in order to reduce the out-gassing).

The back surface of this barrel is designed in order to be mounted on the ESO flange. The Vacuum Barrel is fixed to the ESO Flange via eight holes matching equivalent 8 threaded (M4) holes in the ESO Cryostat flange.

The Spectrographs Cameras will be mounted onto the interface flange of the cryostat. This to simplify the optical alignment between the main camera optical train and the last lens which act as Cryostat window and is hence part of the Cryostat itself.

The movable part of the camera has been ordered at the optical vendor mounted in a barrel accommodating the focusing mechanism and moved by a SD40-2 servo unit provided by the Consortium. It is the responsibility of the vendor to design the mechanical layout of this system according to the specification of volumes and connections given by the Consortium. The connection will have to be on the Vacuum Barrel (see Figure 14) for which 8 threaded holes (M4) have been reserved

\section{THE FINITE ELEMENT MODEL}

Finite Element Modeling (FEM) has been an essential tool to control the amount of image movement and distortion caused by gravitational flexure and temperature drift. In stead of analyzing a detailed FE-model of the complete instrument, the analysis has been split into modules. This has allowed several teams to study the instrument in parallel, and greatly eased the implementation of changes. This also allowed each team to work with their preferred FEA tool. The results from the individual modules have then been numerically combined, where required. The gravitational flexure has been charted by calculating the deformation for a Zenith Distance (ZD) of 60 degrees for all rotator angles with a spacing of 30 degrees. The flexure is found by subtracting the deformation at $0^{\circ} \mathrm{ZD}$ from these. The resulting flexure was extracted from points of special interest. These include focal planes and locations of optics surfaces. Image motion sensitivity matrices were calculated for these locations, and by combining these matrices with the flexure data, the resulting image motion could be calculated. The motion was then evaluated against the requirements.

\section{MODEL OF THE BACKBONE AND SPECTROGRAPH BENCHES}


The model described here consists of a detailed model of the backbone structure, including the internal pre-slit optics mounts. The UVB and VIS spectrographs are included in the model, with fully detailed optic benches and enclosures. Simplified models of the supports of the spectrograph optics are included, only consisting of rigid bodies with appropriate mass and mounting interfaces. The model has 25178 nodes and 22502 elements (see Figure 15). The analysis has been performed with MSC. Nastran for Windows.

Detailed simulations of the NIR spectrograph is not included in these simulations. Still the mass, Stiffness and the inertia of NIR has to be considered. This is done by adding the weight of NIR as a mass element which also includes inertia located in the center of gravity. The Center of Gravity node is connected to the backbone interface via a semi rigid body to the interface points. The stiffness between the interface points from NIR is simulated as springs. The adapter flange of the telescope is simulated as an infinitely stiff surface since the stiffness is not known at present. Finally, a simplified model of the support for electronics boxes is included.

This model serves multiple purposes: It provides detailed data for image motion analysis inside the backbone, and it delivers the fundament for a detailed analysis of image motion inside the UVB and VIS spectrographs. The model is also used for evaluating the stress level and eigenfrequencies in the event of an earthquake.

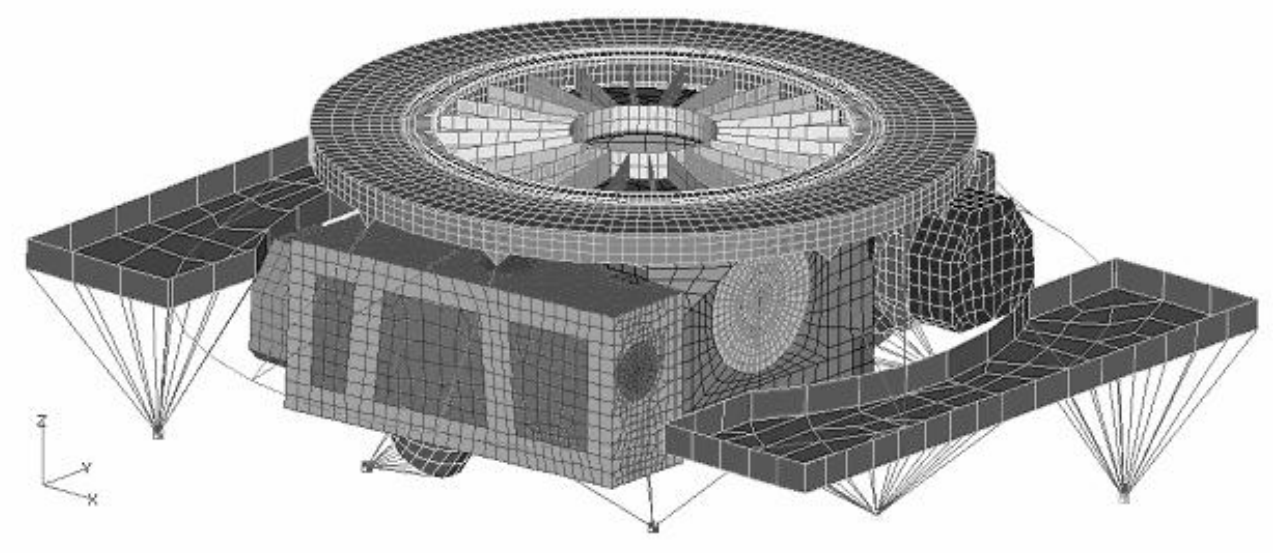

Figure 15 Finite Element Model of the X-shooter. Different shades indicate different plate thickness.

The image in the Cassegrain focal plane is split into the wavelength bands for the three spectrographs, and is re-imaged onto the slits of each spectrograph. To ensure that all three spectrographs are effectively observing the same patch of sky and to minimize the loss of light, very tight requirements of co-alignment of the slits has been set: With the instrument at Zenith, the projected slit positions of the UVB and NIR spectrographs shall coincide with the projected position of the VIS slit:

- to 0.05 " perpendicular to slit direction, at any position along the slit

- to 0.2 " along the slit direction, at the slit ends

The operational conditions are: $0^{\circ}$ to $60^{\circ} \mathrm{ZD}$, a temperature between $0^{\circ}$ and $15^{\circ}$ Celcius and a maximum rate of change of $0.5^{\circ} \mathrm{C} /$ hour with $\mathrm{PV}$ of $5^{\circ} \mathrm{C} /$ night. With these conditions, the maximum drift of each slit projected onto the Cassegrain focal plane must be:

- $<0.08$ " perpendicular to the slit (goal 0.04")

- $<0.2$ " in slit direction

The locations where FEA data were extracted for the image motion model included the cassegrain focus, slit positions, dichroics, folding mirrors and ADCs. An example of the results is given in Figure 16, where the image motion relative to the position of the slit of the VIS spectrograph is displayed. The resulting motion is 0.015 arcsec along the slit, or $8 \%$ of the specification of 0.2 arcsec. Across the slit, the motion is 0.026 ", or $33 \%$ of the specification of 0.08 arcsec and $66 \%$ of the goal of 0.04 arcsec. Similarly, the specifications were met for the VIS arm. However, other factors contribute to the image motion, in particular there is a concern about image wobble caused by the rotating ADC. As a safeguard, 
Active Flexure Compensation was introduced, where the folding mirror in each preslit arm can be tilted by piezo actuators.

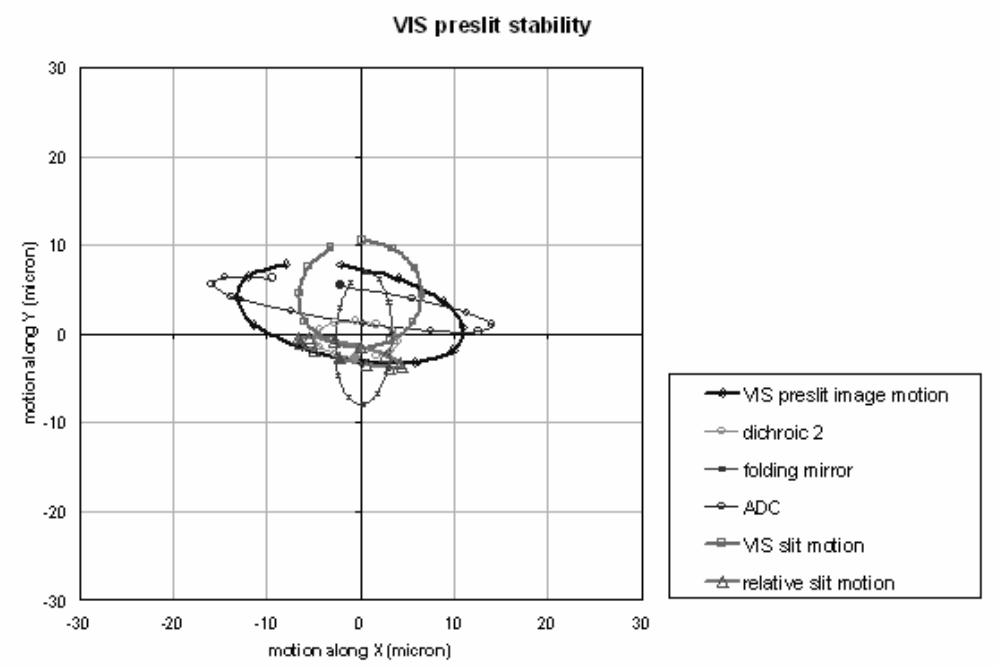

Figure 16 VIS spectrograph slit motion relative to the image for all rotator angles at $Z D=60^{\circ}$. One arcsec corresponds to $254 \mu \mathrm{m}$. The slit is oriented along the $\mathrm{Y}$ direction. All contributors are shown and the total image motion is given by the dark, bold ellipse.

An earthquake analysis was performed, where the model was subjected to $3 \mathrm{G}$ accelerations. Stress levels were satisfactory, with a safety factor better than 1.5 in all cases. Eigenfrequencies for the backbone and spectrographs were also within specifications, as the lowest resonance occurred above $100 \mathrm{~Hz}$. However, the electronics racks did show unacceptable low eigenfrequencies and has subsequently been re-designed to meet specifications.

Similarly for the spectrograph benches, FEA deformations were extracted at the locations of mounting surfaces for optics holders and at the entrance slit and focal plane. Assuming rigid optics mounts, this allowed us to make a first estimate of the image motion. Later, this analysis was refined by including the actual deformation of the mounts, as described below.

\section{THE MODEL OF THE INTERNAL SPECTROGRAPH PARTS}

Two similar models for the VIS and UV-B subunits has been prepared. The subsystems modeled are:

- Main Mirror;

- Combo Unit;

- Pupil Mirror;

- Corrector Lens.

The optical elements are modeled as rigid elements, where the master node is the optical reference point (defined by optical designer), linked to slave nodes: the mass element and the connection points to the supports. This is sufficient since the solid parts are modeled using tetraedrical elements (CTETRA). The connection between the solids with different material are obtained with Constraint Equations, which links the DOFs of the node in the adjacent surfaces. The optical mass characteristics are modeled using Mass Elements in the Center of Gravity of the glasses. Rigid elements was used to model the spherical joint between the shaft and the pad. The master node is in the center of the joint and the slaves are on the pin ring of the "adaptable support". The DOFs locked are only the translational ones. The rigid elements are also used for the optics and for the constraint points. it is only in the displacements of the optical points which is of interest. 

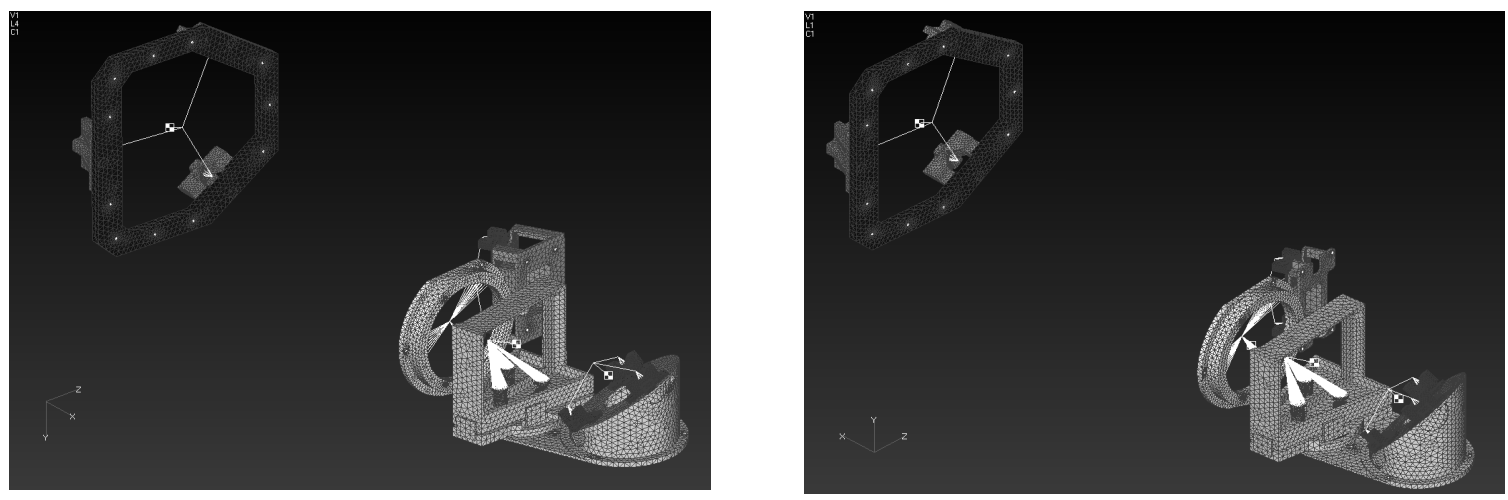

Figure 17 Some elements of the VIS (27353 nodes - 78508 elem.) and the UV-B (25132 nodes - 71231 elem.) subsytems .

\section{CONCLUSIONS}

In this paper we have presented the X-Shooter Spectrograph for 2nd Generation VLT instruments, a result of a collaborative effort of a Consortium formed between ESO and different partner institutes in Italy, Denmark, France and the Netherlands. Four instruments concepts were quoted in the Call for $2^{\text {nd }}$ Generation VLT instruments as being of particular interest to the ESO community: a cryogenic multi-object Spectrometer in the 1 to $2.4 \mu \mathrm{m}$ range, a wide-field 3D optical Spectrometer, a high contrast, adaptive optics assisted, Imager (Planet Finder) and a medium resolution, wideband $(0.32-2.4 \mu \mathrm{m})$ Spectrometer (X-shooter). After interaction with the ESO Scientific and Technical Committee, the study was presented in final form to ESO in October 2003 and fully approved in December 2003. This paper is mainly based on the results of the Final Design Review (February 2006). The project is now in fully carrying out with the a target installation date in the 1Q of 2008.

\section{REFERENCES}

1. X-shooter: ESO/FDR internal Document XSH-TRE-ITA-4000-0053 - Mechanical Design Report - UV-B and VIS Spectrographs dated 07.02.2006.

2. X-shooter: ESO/FDR internal Document XSH-ICD-DMK-2000-0036 - ICD Backbone - Subsystems dated 07.02.2006.

3. X-shooter: ESO/FDR internal Document XSH-ICD-ITA-4000-0054 - ICD UVB \& VIS Spectrographs - CCD Detector System dated 07.02.2006.

4. X-shooter: ESO/FDR internal Document XSH-TRE-ITA-2700-0027 - Optical Design Report dated 07.02.2006.

5. X-shooter: ESO/FDR internal Document XSH-TRE-DMK-2100-0034 - Performance Analysis - FEA dated 07.02.2006.

6. X-shooter: UV-VIS-J+H band, Intermediate Resolution, High Efficiency Spectrograph for the VLT, Part 1- Science Case prepared by the Instrument Science Team, ESO/STC Document 324a, dated 20.03.2003.

7. X-shooter: UV-VIS-J+H band, Intermediate Resolution, High Efficiency Spectrograph for the VLT, Part 2Feasibility Study. ESO/STC Document 324b, Issue 2 dated 06.10.2003.

8. X-shooter: UV-VIS-J+H band, Intermediate Resolution, High Efficiency Spectrograph for the VLT, Part 1- Science Case prepared by the Instrument Science Team, ESO/STC Document 324a, dated 20.03.2003.

9. S. D'Odorico, B. Delabre, H. Dekker, Plans and Studies at ESO for VLT optical Instrumentation, ESO Conference and Workshop, 1988 Proc. 30, 1003.

10. B. Delabre, H. Dekker, F. Merkle, S. D'Odorico, Astronomical Spectrograph with Collimator Compensation of Camera Chromatism (4C),1989, Proc. SPIE Vol. 1055. 